\title{
MiR-34c acts as a tumor suppressor in non-small cell lung cancer by inducing endoplasmic reticulum stress through targeting HMGBI
}

This article was published in the following Dove Press journal: OncoTargets and Therapy

\author{
Li Tu ${ }^{1,2, *}$ \\ Xiang Long ${ }^{2}$ \\ Weidong Song ${ }^{2}$ \\ Zhongdong Lv ${ }^{2}$ \\ Huadong Zeng' \\ Tiezhu Wang ${ }^{3}$ \\ Xianglu Liu $^{2}$ \\ Juanni Dong ${ }^{2}$ \\ Ping $X \mathrm{u}^{2, *}$ \\ 'Department of Respiratory Medicine, \\ Shenzhen Hospital, Southern Medical \\ University, Shenzhen 5I8000, People's \\ Republic of China; ${ }^{2}$ Department of \\ Respiratory Medicine, Peking University \\ Shenzhen Hospital, Shenzhen 5I8000, \\ People's Republic of China; ${ }^{3}$ Department \\ of Respiratory Medicine, Zhangzhou \\ Municipal Hospital of Fujian Province, \\ Zhangzhou 363000, People's Republic of \\ China
}

*These authors contributed equally to this work
Correspondence: Ping $\mathrm{Xu}$

Department of Respiratory Medicine, Peking University Shenzhen Hospital,

\#I I 20 of Lianhua Road Futian Area,

Shenzen 518000, Guangdong, People's

Republic of China

Tel +86 I35 I033 I460

Email pkpingxu@।63.com
Objective: To investigate the role of miR-34c in lung cancer.

Methods: The levels of microRNA-34c (miR-34c) expression in non-small cell lung cancer (NSCLC) tissue and cell lines were examined by the qRT-PCR assay. High mobility group box 1 (HMGB1) expression in NSCLC was assessed by immunohistochemical analysis (IHC), qRT-PCR, and Western blot assays. The effects of miR-34c overexpression or HMGB1 knockdown on cell proliferation and apoptosis were evaluated by CCK- 8 and flow cytometry analysis, respectively. Cellular reactive oxygen species (ROS) production in NSCLC cells was detected using a ROS kit. The levels of Bax, p-ERK, eIF2 $\alpha$, GADD153, and IRE1 $\alpha$ expression in treated NSCLC cells were measured by Western blot assays. In addition, the interaction between miR-34c and HMGB1 was verified by the dual-luciferase reporter assay.

Results: miR-34c was only slightly expressed, while HMGB1 was highly expressed in NSCLC tissues and cell lines. Overexpression of miR-34c or knockdown of HMGB1 inhibited cell proliferation, promoted cell apoptosis, and induced ER stress in NSCLC cells. In terms of mechanism, miR-34c negatively regulated HMGB1 expression by directly targeting the 3 '-untranslated region (UTR) of HMGB1 mRNA. In addition, we proved that HMGB1 overexpression could block the effects of miR-34c on NSCLC cell proliferation, apoptosis, and ER stress.

Conclusion: miR-34c may suppress NSCLC tumors by targeting HMGB1 mRNA, promoting endoplasmic reticulum stress, and increasing ROS levels. Our findings suggest that miR$34 \mathrm{c}$ has a role in NSCLC.

Keywords: non-small cell lung cancer, microRNA, high mobility group box 1, endoplasmic reticulum stress, ER stress

\section{Background}

Although novel therapeutic strategies based on targeted therapy and new compounds have been developed and applied in clinical settings, lung cancer remains one of the most common and fatal malignancies worldwide. The 5-year survival rate of lung cancer patients is generally $<15 \% .{ }^{1}$ More than $80 \%$ of all lung cancers are non-small cell lung cancer (NSCLC), which is the most common subtype of lung cancer, and the majority of NSCLC patients present with symptoms during the advanced stages of their disease. ${ }^{2,3}$ Furthermore, $>50 \%$ of newly diagnosed NSCLC patients already have distant metastasis at the time their diagnosis is confirmed, and the majority of NSCLC-related deaths are caused by metastatic disease. ${ }^{4,5}$ Although 
many improvements have been made in treating NSCLC during past decades, tumor metastasis remains the biggest challenge in its clinical therapy, and until relatively recently, NSCLC patients with advanced stage disease have been considered to be incurable. ${ }^{6,7}$ Therefore, it is essential to better understand the mechanisms of NSCLC tumorigenesis and identify novel therapeutic targets and diagnostic biomarkers for NSCLC.

MicroRNA (miRNA) is widely expressed in mammalian cells, and is a critical RNA molecule that was first identified when studying heterochronic mutants of Caenorhabditis elegans by use of genetic methods. Numerous studies have shown that various miRNAs are expressed in time-dependent and tissue-specific manners. ${ }^{8}$ Moreover, the expression profiles of miRNAs are dysregulated in multiple human cancers, including NSCLC, liver cancer, breast cancer, and gastric cancer. These findings suggested that miRNAs could be utilized as unique biomarkers for tumor progression. The miR-34 family includes three types: miR-34a, miR-34b, and miR-34c. MiR-34a originates from its own transcript, while miR$34 \mathrm{~b}$ and miR-34c share a common primary transcript. ${ }^{9}$ In mice, miR-34a was found to be ubiquitously expressed in the brain, while miR-34b and miR-34c were mainly present in lung tissue. ${ }^{9}$ Recently, miR-34c was revealed to be involved in various human diseases, including neuropathic pain, diabetic corneal neuropathy, and cancers. ${ }^{10-12}$ However, its role in NSCLC remains largely unclear.

Endoplasmic reticulum (ER) is an important organelle involved in lipid and protein synthesis. Its function can be disturbed by various stimuli, such as hypoxia, dysfunctional protein synthesis, and calcium overload; these disturbances can result in ER stress. ${ }^{13}$ Previous studies have shown that ER stress plays a critical role in regulating tumor cell apoptosis by activating multiple ER-stress associated proteins, such as inositol requiring protein1 $\alpha$ (IRE1 $\alpha$ ), PKR-like ER kinase (PERK), eukaryotic translation initiation factor (eIF), and GADD153. ${ }^{14}$ High mobility group box 1 (HMGB1) is a nuclear DNA-binding protein that comprises 215 amino acid residues and has three distinct domains: two tandem HMG box domains and an acidic C-terminal tail of 30 amino acids. ${ }^{15}$ HMGB1 was previously reported to participate in the pathogenesis of various human diseases, such as sepsis, ${ }^{16}$ and cancers, ${ }^{17}$ by interacting with miRNAs. During ER stress, neurons could release HMGB1 to trigger the initiation of neuron-inflammation and glial activation. ${ }^{18}$ As for cancer research, HMGB1 has been demonstrated as an oncogene inhibiting cell apoptosis through mediating ER stress, ${ }^{19}$ accompanying with reactive oxygen species (ROS) production. ${ }^{20}$ A recent study suggested that ROS induction can lead to cell apoptosis and exert a anti-drug-resistance effect on lung cancer. ${ }^{21}$ This might be due to the occurrence of DNA damage in lung cancer induced by ROS generation through mitochondrial membrane potential reprogramming. ${ }^{22-24}$

In the present study, we investigated the effects of miR-34c and HMGB1 on NSCLC growth and ER stress, as well as the association between miR-34c and HMGB1. This was done to better understand the pathogenesis of NSCLC, and identify several novel therapeutic targets for NSCLC.

\section{Materials and methods NSCLC tissue samples and cell lines}

A total of 20 pairs NSCLC and adjacent normal tissue samples were collected from Peking University Shen Hospital during 2014-2019. Written informed consent was obtained from each subject in advance, and the study protocol was approved by the Ethics Committee of Peking University Shen Hospital. The normal human lung cell line (MRC-5) and five human NSCLC cell lines (A549, H460, H157, H1299, and H23) were purchased from the Type Culture Collection of the Chinese Academy of Sciences (Shanghai, China). The cells were maintained at $37{ }^{\circ} \mathrm{C}$ in RPMI-1640 medium (HyClone Laboratories Inc., Logan, UT, USA) containing 10\% fetal bovine serum (FBS), $1 \%$ penicillin/streptomycin, in an atmosphere of $5 \%$ $\mathrm{CO}_{2}$ and $95 \%$ air.

\section{Immunohistochemical analysis (IHC)}

Briefly, fixed samples of NSCLC and normal tissue were embedded with paraffin and then sliced into $4 \mu \mathrm{m}$ sections. Next, the tissue sections were deparaffinized, dehydrated, and their antigens were retrieved. The slices were then incubated in $3 \% \mathrm{H}_{2} \mathrm{O}_{2}$ for $1 \mathrm{~h}$, followed by incubation in $10 \%$ donkey serum for $2 \mathrm{~h}$. Next, the slices were stained overnight with a primary antibody against HMGB1 (rabbit, 1:2,000, Abcam, Cambridge, UK), using the streptavidin peroxidase-conjugated (SP-IHC) method.

\section{Hematoxylin/eosin (H\&E) staining}

The treated NSCLC tissue samples were stained with Harris hematoxylin (Surgipath, Richmond, IL, USA), acid alcohol ( $1 \%$ concentrated hydrochloric acid in $70 \%$ ethanol), and $0.1 \%$ eosin (Surgipath) using a Shandon Linistain ${ }^{\mathrm{TM}}$ GLX Linear Stainer (Thermo Scientific, Cheshire, UK). 


\section{RNA extraction and quantitative real- time PCR (RT-PCR)}

The total RNA of NSCLC and normal tissue samples, as well as treated and non-treated NSCLC cells was extracted with TRIzol reagent (Invitrogen, Carlsbad, CA, USA). After determining the quality of the extracted RNA with a NanoDrop2000c spectrophotometer (Thermo Scientific, Waltham, MA, USA), $2 \mu \mathrm{g}$ of total RNA was used as a template for the reverse transcription of cDNA that was performed using a BestarTM qPCR RT Kit (\#2220, DBI Bioscience, China). The RT-PCR was performed using BestarTM qPCR MasterMix (\#2043, DBI Bioscience, China) on an ABI7500 system for purposes of quantifying the levels of miR-34c and HMGB1 in NSCLC tissues and cells. The sequences of the primers used were as follows: GAPDH, F: 5'-TGT TCG TCA TGG GTG TGA AC-3', R: 5'-ATG GCA TGG ACT GTG GTC AT-3'; U6, F: 5'- CTC GCT TCG GCA GCA CA -3', R: 5'-AAC GCT TCA CGA ATT TGC GT-3'; miR-34c, F: 5'- ACACTCCAGCTGGG AGGCAGTGTAGTTAGCTG -3', R: 5'- CTCAACTGGTG TCGTGGA -3', RT: 5'-CTC AAC TGG TGT CGT GGA GTC GGC AAT TCA GTT GAG GCA ATC AG-3'; HMGB1F: 5'-CTC GCT TCG GCA GCA CA-3', R: 5'AAC GCT TCA CGA ATT TGC GT-3'. The level of HMGB1 expression was normalized to that of GAPDH, and miR-34c expression was normalized to that of U6. Gene expression was quantified using the $2^{-\Delta \Delta \mathrm{Ct}}$ method.

\section{Western blot assay}

The total proteins of NSCLC tissues and cells were extracted using a radioimmunoprecipitation assay reagent that contained protease inhibitors (Beyotime Institute of Biotechnology, Haimen, China). After determining the concentration of protein in each sample with a BCA Protein Assay Kit (Beyotime Institute of Biotechnology), $40 \mu \mathrm{g}$ of denatured total protein was separated by $10 \%$ SDS-PAGE. Next, the target proteins were transferred onto nitrocellulose membranes (EMD Millipore, Billerica, MA, USA), which were subsequently incubated in 5\% low fat milk for $2 \mathrm{~h}$ at room temperature. After incubation, the membranes were washed twice with PBS, and then incubated overnight with primary antibodies against HMGB1, Bax, p-ERK, eIF2 $\alpha$, GADD153, and IRE1 $\alpha$, followed by a $2 \mathrm{~h}$ incubation with peroxidase-conjugated secondary antibodies (anti-mouse, Cat. No. SC-2005 and anti-rabbit, Cat. No. SC-2004). Finally, the membranes were analyzed with an enhanced chemiluminescence detection system (EMD Millipore,
Burlington, MA, USA). The primary antibodies used were anti-HMGB1 (Dilution 1: 1,000, Abcam, ab77302), anti-Bax (Dilution 1: 1,000, Abcam, ab32503), anti-p-ERK (Dilution 1: 500, Abcam, ab76165), anti-eIF2 $\alpha$ (Dilution 1: 1,000, Abcam, ab26197), anti-GADD153 (Dilution 1: 1,000, Abcam, ab11419), anti-IRE1 $\alpha$ (Dilution 1: 100, Abcam, ab37073), and anti-GAPDH (Dilution 1: 2,000, Santa Cruz Biotechnology, Dallas, TX, USA).

\section{Cell counting kit-8 (CCK-8) assay}

The effects of miR-34c and HMGB1 on the proliferation of NSCLC cells were evaluated by the CCK- 8 assay. In brief, $2 \times 10^{4}$ treated NSCLC cells were seeded into the wells of 96well plates, and incubated at $37^{\circ} \mathrm{C}$ for $24 \mathrm{~h}$. Next, the CCK-8 assay was used to assess cell viability at intervals of 24,48 , and $72 \mathrm{~h}$ after seeding. The absorbance at $450 \mathrm{~nm}$ was detected with a microplate reader (Thermo Plate, Rayto Life and Analytical Science, Co., Ltd., Hamburg, Germany).

\section{Cell apoptosis analysis}

The effects of miR-34c and HMGB1 on cell apoptosis were studied using an Annexin V-fluorescein isothiocyanate (FITC) and propidium iodide (PI) apoptosis detection kit (BestBio, Shanghai China), followed by a flow cytometric analysis. Briefly, treated A549 and NCI-157 cells $\left(2 \times 10^{4}\right)$ were harvested and re-suspended in culture medium. After staining with Annexin V FITC/PI according to the manufacturer's instructions, the cells were analyzed by flow cytometry (Becton-Dickenson, Franklin Lakes, NJ, USA).

\section{Detection of cellular ROS production}

The cellular ROS production of treated A549 and NCIH157 cells was detected using a ROS Kit (CA1410, Solarbio Life Science, Beijing, China). In brief, treated NSCLC cells were cultured at $37^{\circ} \mathrm{C}$ for $24 \mathrm{~h}$; after which, they were collected and re-suspended in serum-free culture medium containing $10 \mu \mathrm{mol} / \mathrm{L}$ DCFH-DA at a concentration of $2 \times 10^{7}$ cells $/ \mathrm{mL}$. After incubation at $37^{\circ} \mathrm{C}$ for $20 \mathrm{~min}$, the NSCLC cells were observed with a laser scanning confocal microscope (Leica, Heidelberg, Germany).

\section{Plasmid construction and dual luciferase activity assay}

For the construction of recombinant luciferase reporter plasmids, the wild type (WT) and mutant (Mut) fragments of the 3'-UTR of HMGB1 mRNA containing putative miR-34c binding sites were amplified and inserted into a 
pGL3 (Promega, Madison, WI, USA) vector to create a HMGB1-WT and HMGB1-MUT recombinant plasmid. For the dual-luciferase reporter assay, A549 cells were seeded into 96 -well plates $\left(1 \times 10^{4}\right.$ cells/well $)$ and cultured overnight at $37^{\circ} \mathrm{C}$; after which, they were co-transfected with miR-34c mimics or the miR-NC plus the HMGB1WT or HMGB1-MUT. The firefly and Renilla luciferase activities of the treated A549 cells were examined using a Dual-Luciferase Assay System (Promega), and Renilla luciferase activity was normalized to Firefly luciferase activity.

\section{Statistical analysis}

All data were analyzed using Graphpad software (Ver. Prism 7, GraphPad Prism Software, La Jolla, CA, USA), and results are expressed as the mean \pm SEM. Student's test and one-way analysis of variance were used to analyze differences between groups, and a $P$-value $<0.05$ was considered to be statistically significant. Each experiment was repeated at least three times.

\section{Results}

MiR-34c was downregulated and HMGBI was upregulated in NSCLC

The pathological changes seen in NSCLC tissues were evaluated by $\mathrm{H} \& \mathrm{E}$ staining. When compared to adjacent normal tissues, the structure of NSCLC tissues was obviously destroyed; furthermore, the nuclear chromatin was darker, and the number of tumor cells was significantly increased (Figure 1A). To further investigate the role of HMGB1 in NSCLC, we detected the levels of HMGB1 expression in NSCLC tissues by use of an immunohistochemical staining (IHC) assay. Our results showed that HMGB1 was significantly upregulated in NSCLC tissues (Figure 1B). Moreover, an analysis of 20 paired NSCLC and adjacent normal tissue samples performed using the qRT-PCR assay also showed that the relative level of miR-34c expression was significantly decreased in the NSCLC tissues when compared with the adjacent normal tissues (Figure 1C). An analysis of 10 paired NSCLC and adjacent normal tissue samples performed using qRT-PCR and Western blot assays revealed a significant upregulation of HMGB1 mRNA and protein in the NSCLC tissues when compared with the adjacent normal tissues (Figure 1D and E). In addition, the relative levels of miR-34c expression were remarkably lower in four NSCLC cell lines (A549, H157, H1299, and H23) when compared to their expression levels in the normal human lung cell line (MRC-5) (Figure 1F).

\section{MiR-34c overexpression significantly inhibited cell growth and induced ER stress in NSCLC cells}

The degree of miR-34c overexpression in A549 and NCIH157 cells was assessed using the qRT-PCR assay. Results indicated a significant upregulation of miR-34c in A549 and NCI-H157 cells transfected with miR-34c mimics when compared to those cells treated with the NC (Figure 2A). After transfecting the NSCLC cell lines (A549 and NCI$\mathrm{H} 157$ ) with miR-34c mimics or the negative control (NC), we evaluated the proliferation and apoptosis of those cells by use of CCK-8 and flow cytometric assays, respectively. Results of the CCK- 8 assays suggested that the proliferative ability of both NSCLC cell lines transfected with miR-34c mimics was significantly reduced when compared to the NC group (Figure 2B). Results from flow cytometry analyses revealed that A549 and NCI-H157 cells treated with miR$34 \mathrm{c}$ had a higher rate of apoptosis than cells treated with the $\mathrm{NC}$ (Figure 2C, lower panel). Furthermore, we also investigated the effects of miR-34c overexpression on intracellular ROS production in A549 and NCI-H157 cells by using a ROS Assay Kit. The fluorescence image analysis showed that ROS production in A549 and NCI-H157 cells treated with miR-34c mimics was significantly increased when compared to ROS production in the Blank and NC groups (Figure 2C, upper panel). In addition, a Western blot analysis revealed significantly increased levels of Bax, p-ERK, eIF2 $\alpha$, GADD153, and IRE1 $\alpha$ in A549 and NCI-H157 cells that overexpressed miR-34c, when compared to cells in the $\mathrm{NC}$ group (Figure 2D). Bax is an apoptosis-related protein, and p-ERK, eIF2 $\alpha$, GADD153, and IRE1 $\alpha$ are associated with the endoplasmic reticulum stress (ERS) mechanism. Our findings suggest that miR-34c overexpression significantly inhibited NSCLC cell proliferation, and promoted NSCLC cell apoptosis and ER stress.

\section{MiR-34c negatively regulated HMGB I expression in NSCLC cells}

To demonstrate the regulatory effect of miR-34c on HMGB 1 expression, we identified a putative miR-34c binding site located in the $3^{\prime}$-UTR of HMGB1 mRNA by performing a bioinformatics analysis (Figure $3 \mathrm{~A}$ ). We then used the dualluciferase reporter assay to confirm the interaction between miR-34c and HMGB1 in A549 cells. Our results showed that 

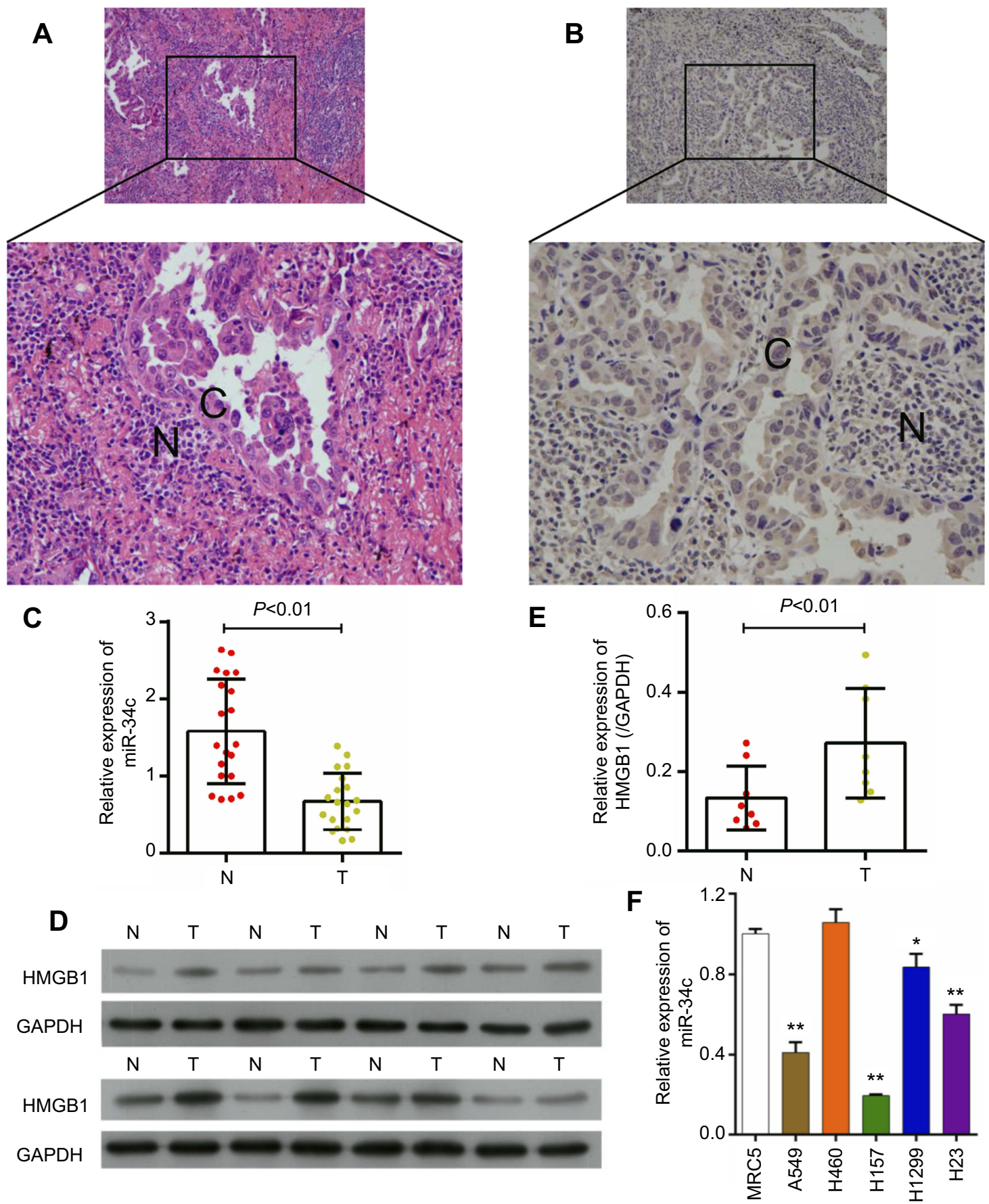

Figure I miR-34c expression was significantly decreased, while HMGBI expression was significantly increased in NSCLC. (A) Examples of NSCLC tissues stained with H\&E. Magnification, $\times 200$, bar $=50 \mu \mathrm{m}$. (B) HMGBI expression was assessed by IHC assays performed with a specific HMGBI antibody. Magnification, $\times 200$, bar $=50 \mu \mathrm{m}$. (C) The relative levels of miR-34c expression in NSCLC and corresponding normal tissues were examined by the qRT-PCR assay. (D and E) The relative levels of HMGBI protein and mRNA expression in NSCLC and corresponding normal tissues were examined by Western blot and qRT-PCR assays, respectively. (F) The levels of miR-34c in one normal human lung cell line (MRC-5) and five human NSCLC cell lines (A549, H460, HI57, HI299, and H23), $* P<0.05$, $* * P<0.0 \mathrm{I}$ vs MRC5 cells.

Abbreviation: NSCLC, non-small cell lung cancer

when compared to the $\mathrm{NC}$ group, miR-34c mimics could significantly attenuate the luciferase activity of A549 cells driven by HMGB1-WT, but not by HMGB1-MUT (Figure 3B). To further assess the effect of miR-34c on HMGB1 expression, we performed Western blot assays to examine the levels of HMGB1 protein expression in A549 and NCIH157 cells transfected with miR-34c mimics or the NC. Results showed that HMGB1 levels were significantly lower in the miR-34c-transfected A549 and NCI-H157 cells when compared to cells in the NC group (Figure 3C). 
A

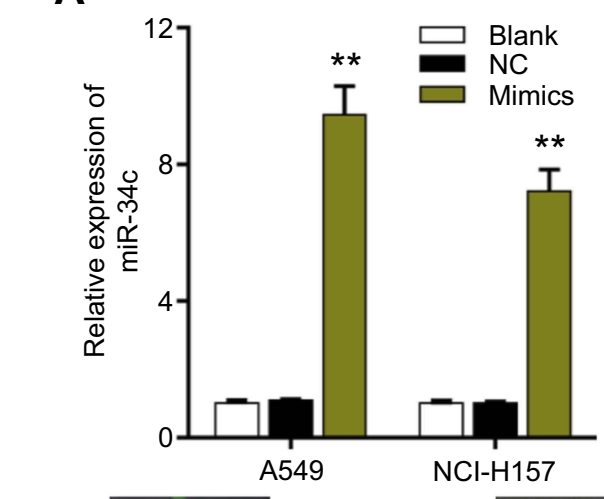

B

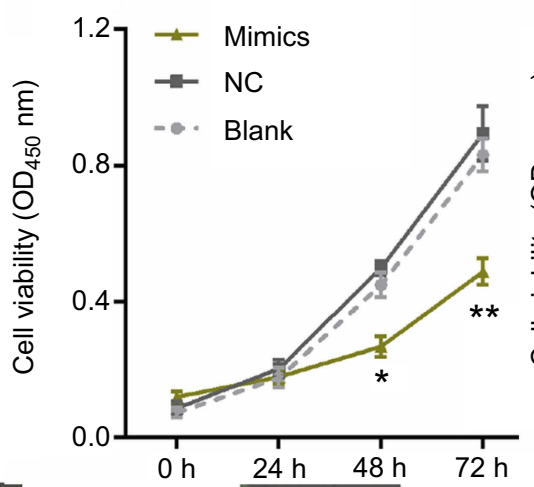

\section{C}
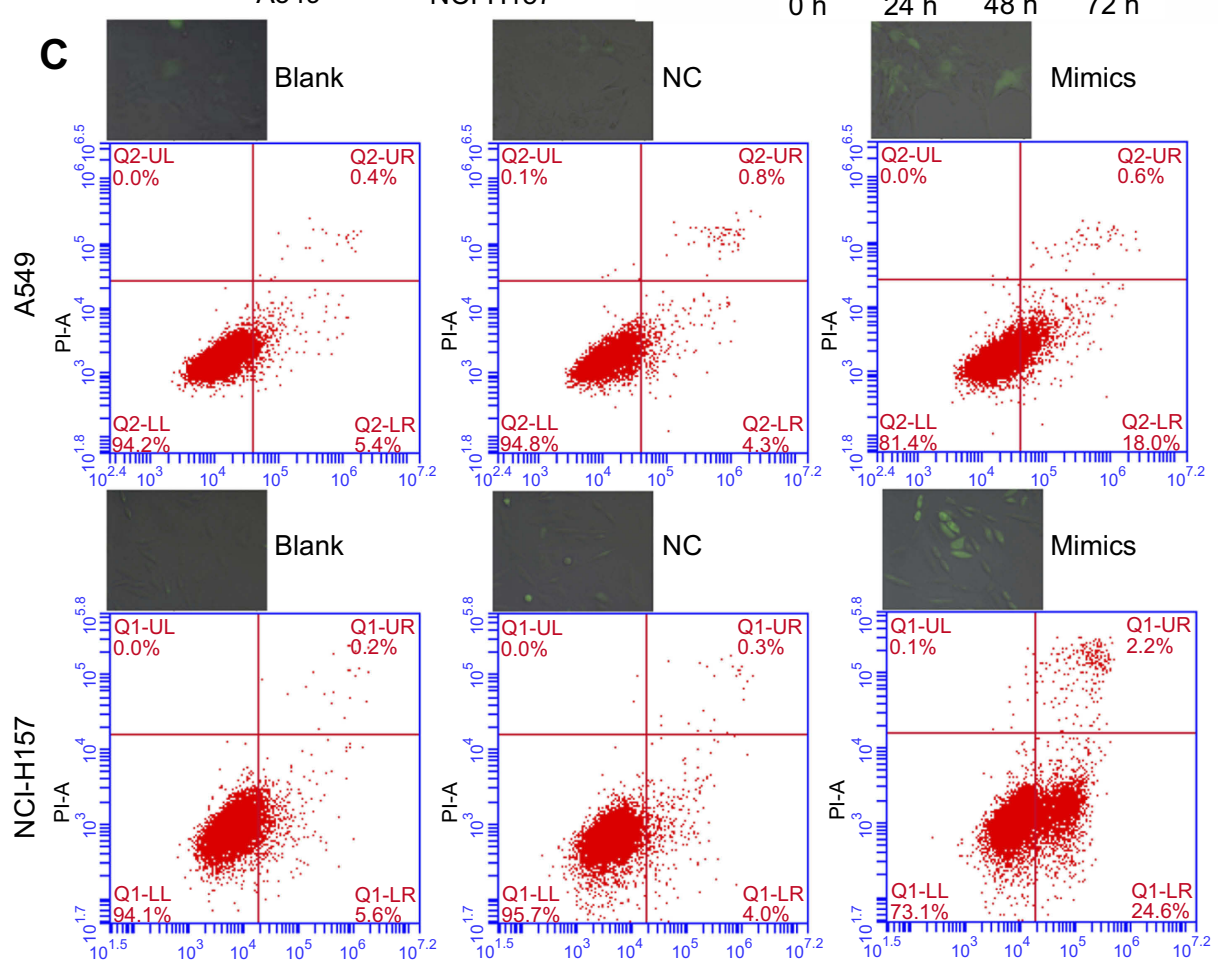

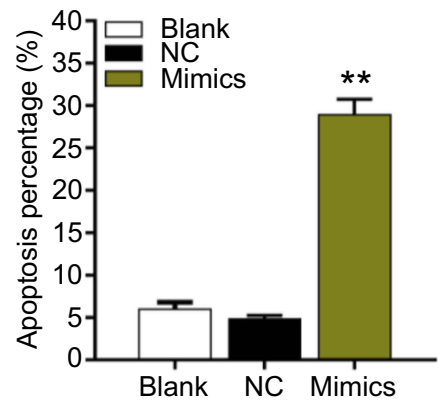

$\mathrm{NCl}-\mathrm{H} 157$
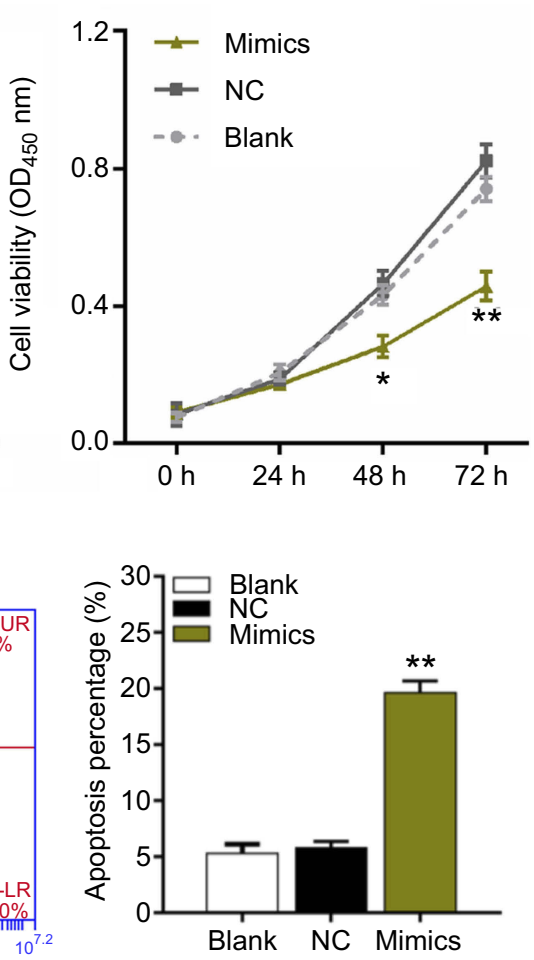

Blank NC Mimics

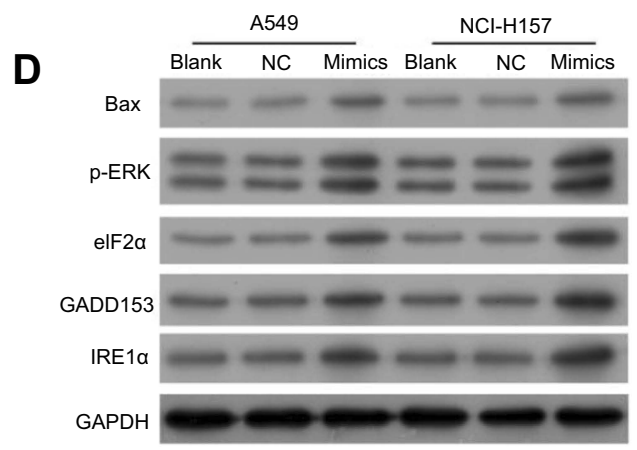

Figure 2 miR-34c overexpression significantly inhibited proliferation, promoted apoptosis and induced endoplasmic reticulum stress in non-small cell lung cancer cells. (A) The relative levels of miR-34c expression in $\mathrm{A} 549$ and $\mathrm{NCl}-\mathrm{HI} 57$ cells transfected with nothing (blank control), the miR-34c negative control (NC), and miR-34c mimics were measured by qRT-PCR assays, ${ }^{* * P}<0.01$ vs NC group. (B) Effects of miR-34c on the proliferation of $A 549$ and NCI-HI 57 cells were evaluated via the CCK-8 assay, $* P<0.05$, $* * P<0.01$ vs $\mathrm{NC}$ group. (C) upper panel: cellular reactive oxygen species production was detected in A549 and NCl-HI57 cells transfected with nothing, miR-34c, the NC or miR-34c mimics. Lower panel: flow cytometry analyses were performed to assess the apoptosis rates of A549 and NCl-HI57 cells transfected with nothing, miR-34c, the NC or miR-34c mimics, **P $<0.0$ I vs NC group. (D) The levels of Bax, p-ERK, elF2 $\alpha$, GADDI53, and IREI $\alpha$ in miR-34c-treated A549 and NCI-HI57 cells were examined via Western blot assays. 


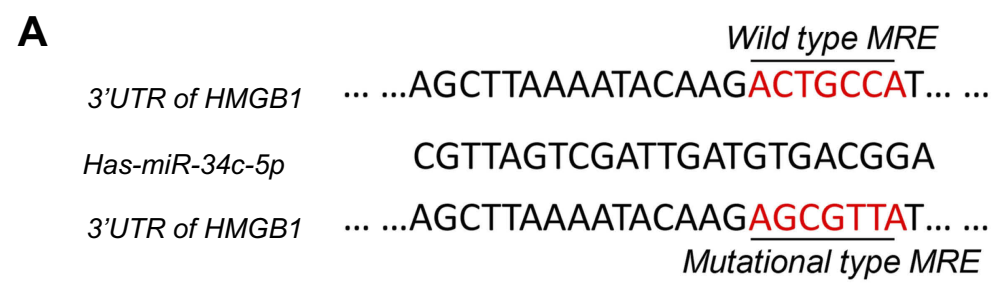

B

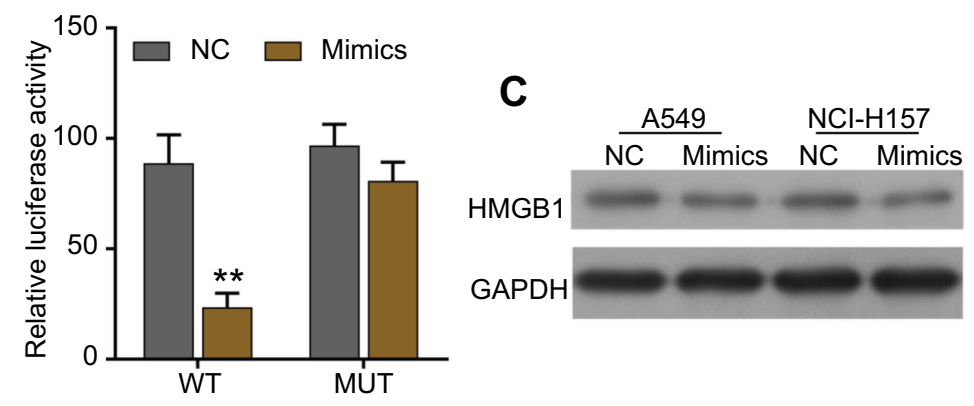

Figure 3 miR-34c negatively regulated HMGBI expression in non-small cell lung cancer cells by directly binding to HMGBI. (A) The putative binding sites between the ${ }^{\prime}$ UTR of HMGBI and miR-34c. (B) Interaction between HMGBI and miR-34c was verified by the dual-luciferase reporter assay. (C) The levels of HMGBI expression in A549 and NCl-HI57 cells transfected with miR-34c, the NC or mimics were measured by Western blot assays.

\section{Knockdown of HMGBI strongly inhibited NSCLC cell growth and promoted ER stress}

To investigate the role of HMGB1 in NSCLC, HMGB1 expression was silenced by using two siRNAs against HMGB1 (si-HMGB1-1 and si-HMGB1-2) in vitro. The knockdown efficiency of the siRNAs was assessed via the qRT-PCR (Figure 4A). Subsequently, the effects of HMGB1 knockdown on cell proliferation and apoptosis were evaluated via CCK-8 and flow cytometry analysis, respectively. Results from CCK-8 assays indicated that both NSCLC cell lines transfected with si-HMGB1-1 and si-HMGB1-2 showed a significant inhibition of cell proliferation when compared with cells in the $\mathrm{NC}$ group (Figure 4B). A flow cytometric analysis of cell apoptosis showed that knockdown of HMGB1 significantly promoted the apoptosis of A549 and NCI-H157 cells (Figure 4C, lower panel; Figure 4D). We then investigated the effects of HMGB1 knockdown on ROS production in A549 and NCI-H157 cells. Results from fluorescence images indicated that ROS production in A549 and NCIH157 cells transfected with si-HMGB1 and si-HMGB2 was significantly higher than in A540 and NCI-H157 cells transfected with nothing or the NC (Figure 4C, upper panel). Furthermore, Western blot assays showed that HMGB1 knockdown resulted in a significant upregulation of Bax, p-ERK, eIF2 $\alpha$, GADD153, and IRE1 $\alpha$ expression in both A549 and NCI-H157 cells (Figure 4E).

\section{HMGBI overexpression reversed the effects of miR-34c on NSCLC cell growth and ER stress}

To better understand the association between HMGB1 and miR-34c in NSCLC cells, miR-34c mimics and HMGB1 plasmids were co-transfected into A549 and NCI-H157 cells, followed by the detection of NSCLC cell proliferation, apoptosis, and ROS production. Results from qRT-PCR assays showed that the downregulation of HMGB1 levels in A549 and NCI-H157 cells induced by miR-34c mimics could be reversed by the ectopic expression of HMGB1 (Figure 5A). CCK-8 assays revealed that A549 and NCIH157 cell proliferation was suppressed by miR-34c mimics, whereas the ectopic expression of HMGB1 could abrogate the inhibitory effect of miR-34c on cell proliferation (Figure 5B). Moreover, our flow cytometric analysis showed that the promotive effect of miR-34c mimics on A549 and NCI-H157 cell apoptosis could be partially reversed by the ectopic expression of HMGB1 (Figure 5C, lower panel, Figure 5D). An analysis of cellular ROS production revealed that HMGB1 overexpression could block the increased production of ROS in A549 and NCI-H157 cells induced by miR$34 \mathrm{c}$ transfection (Figure 5C, upper panel). In addition, results from Western blot analyses suggested that miR-34c mimics caused an upregulation of Bax, p-ERK, eIF2 $\alpha$, GADD153, and IRE1 $\alpha$ expression in A549 and NCI-H157 cells, and that effect was also abolished by the ectopic expression of HMGB1 (Figure 5E). 


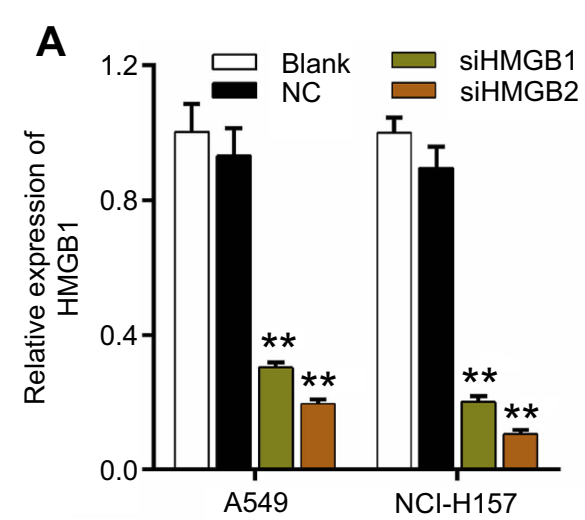

B

A549

$\mathrm{NCl}-\mathrm{H} 157$
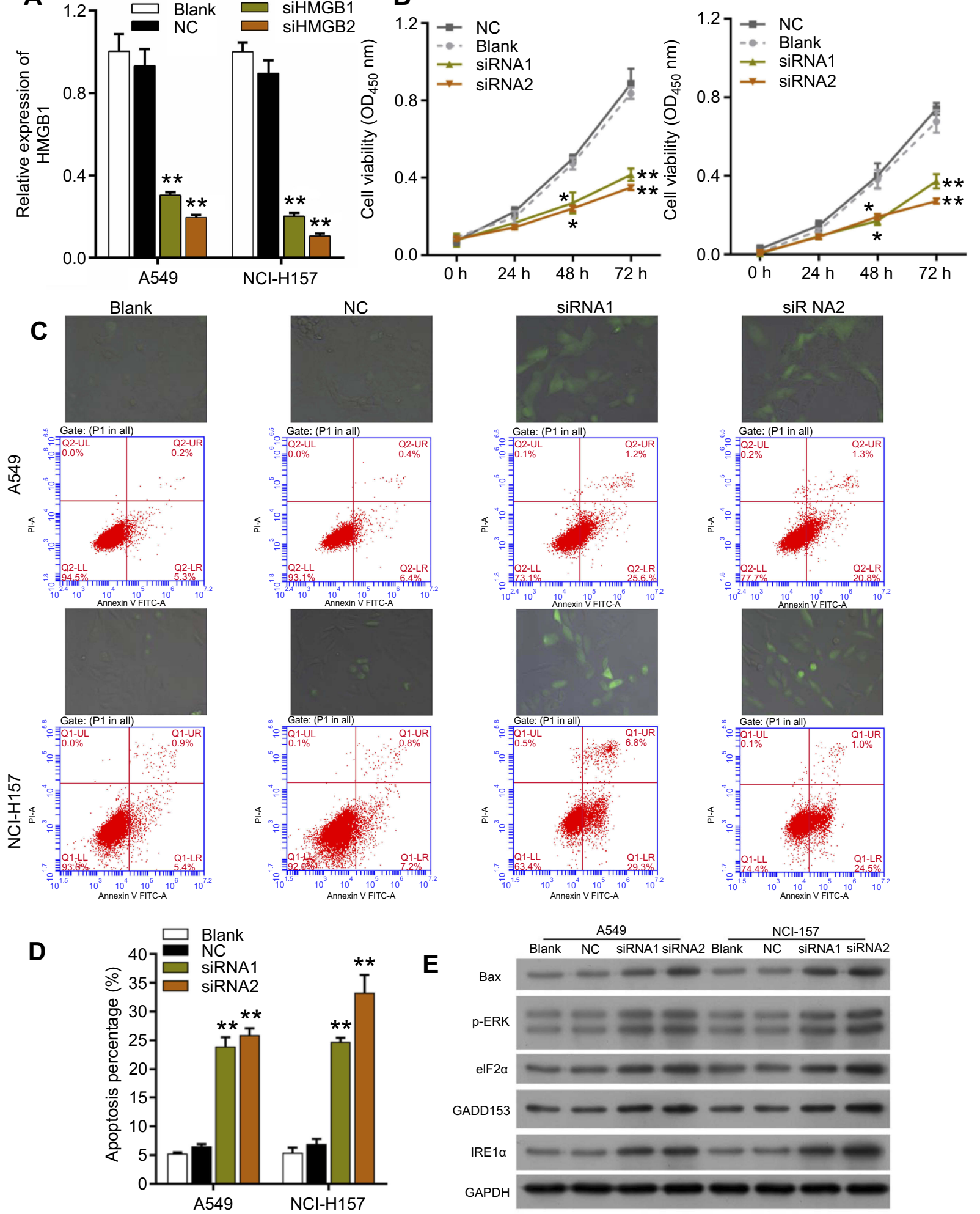

Figure 4 Knockdown of HMGBI strongly inhibited proliferation and promoted apoptosis and endoplasmic reticulum stress in non-small cell lung cancer cells. (A) The knockdown efficiencies of two siRNAs targeting HMGBI (si-HMGBI-I and si-HMGBI-2) in A549 and $\mathrm{NCl}-\mathrm{HI} 57$ cells were assessed via the qRT-PCR assay, **P<0.0I vs $\mathrm{NC}$ group. (B) The effect of treatment with si-HMGBI-I and si-HMGBI-2 on the proliferation of A549 and NCI-HI57 cells was evaluated via the CCK-8 assay, **P<0.0I vs NC group. (C and D) Upper panel: the effects of HMGBI knockdown on cellular reactive oxygen species (ROS) production in A549 and $\mathrm{NCl}-\mathrm{HI} 57$ cells transfected with nothing, the $\mathrm{NC}$ or si-HMGBI-I and si-HMGBI-2 were analyzed by using a ROS kit. Lower panel: the apoptosis rates of A549 and $\mathrm{NCl}-\mathrm{HI} 57$ cells transfected with nothing, the NC or si-HMGBI-I and si-HMGBI-2 were detected by flow cytometric analysis (D), ${ }^{* * P<0.0 I}$ vs NC group. (E) Western blot analysis of Bax, $\mathrm{P}$-ERK, elF2 $\alpha$, GADDI53, and IREI $\alpha$ expression in HMGBI-blocked A549 and $\mathrm{NCl}$ - I57 cells. 
A

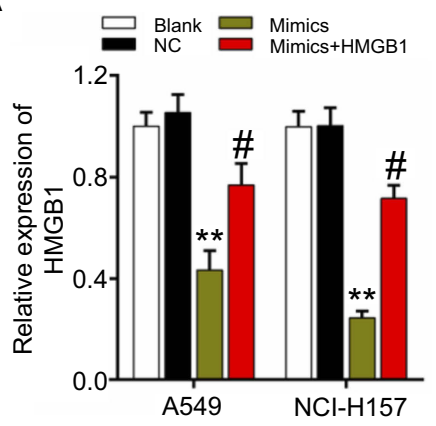

B

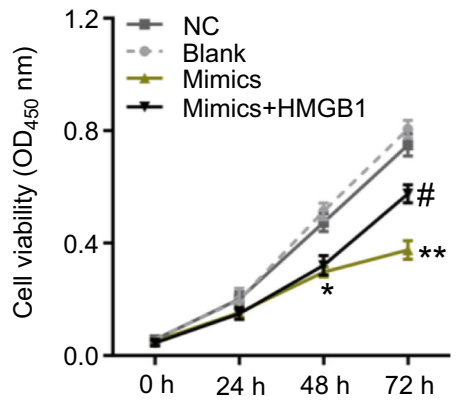

NCl-H157

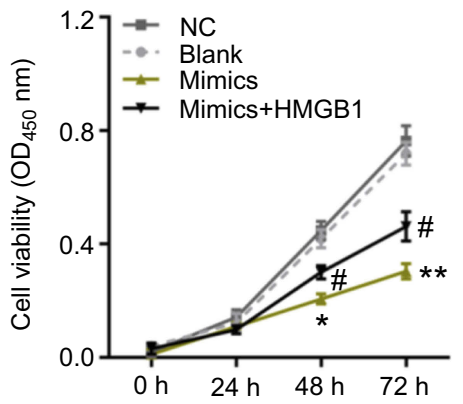

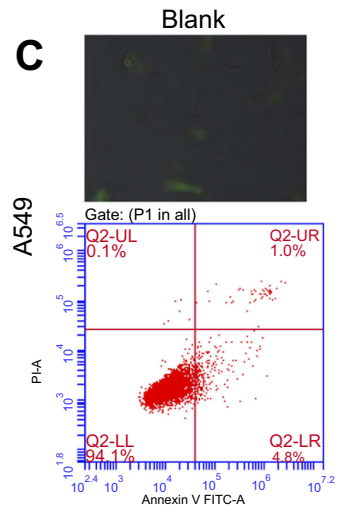
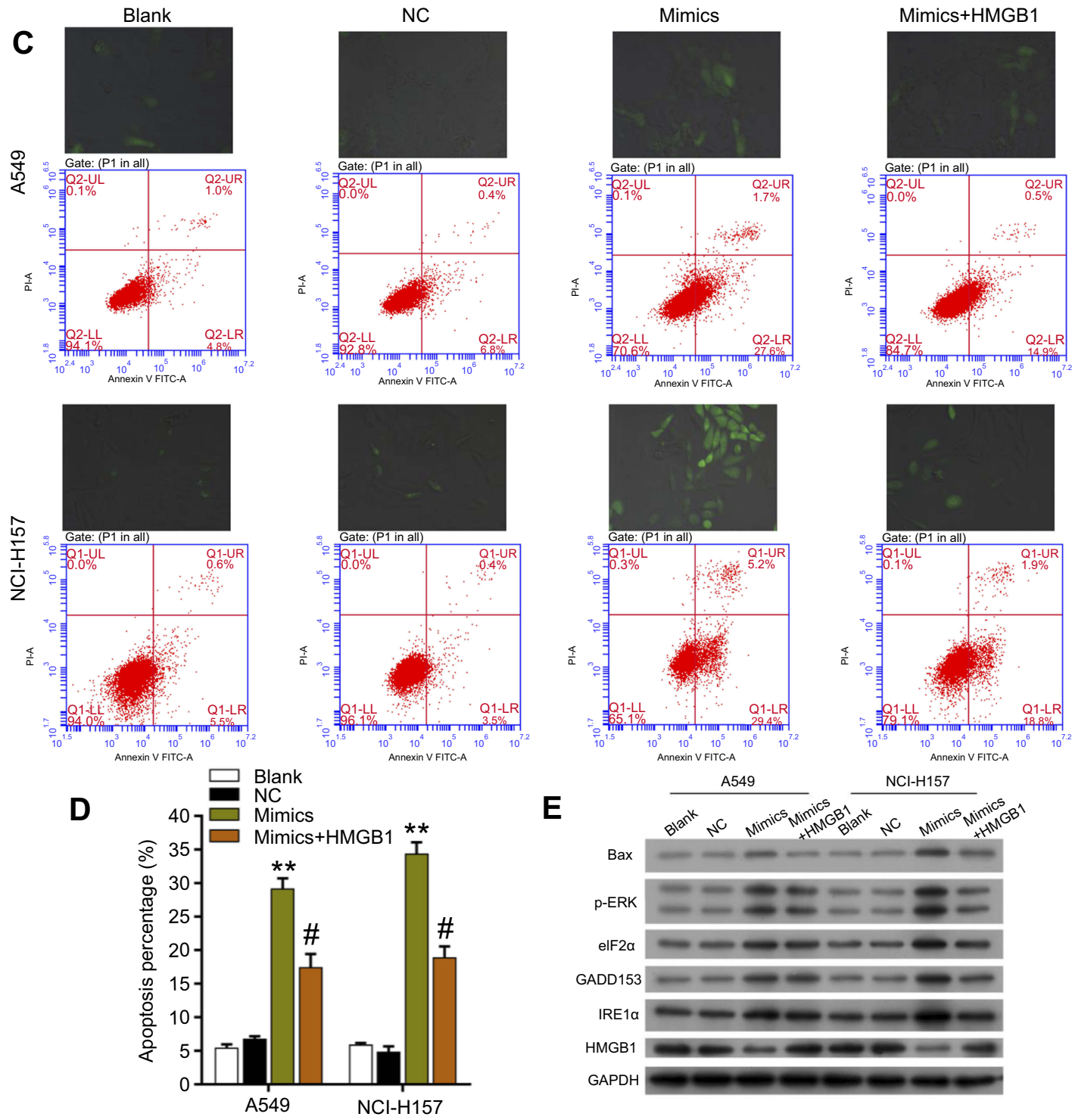

E

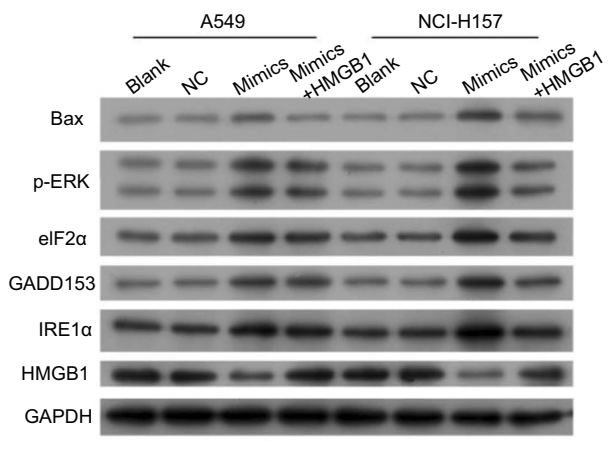

Figure 5 MiR-34c suppressed proliferation, accelerated apoptosis, and alleviated endoplasmic reticulum stress in non-small cell lung cancer cells via its effect on $\mathrm{HMGBI}$. (A) qRT-PCR analysis of HMGBI expression in A549 and NCl-HI57 cells treated with nothing, the NC, miR-34c mimics, or miR-34c mimics $+\mathrm{HMGBI}$, $* * P<0.01$ vs NC group, $\# \mathrm{P}<0.05$ vs mimics. (B) CCK-8 analysis of the proliferation of $\mathrm{A} 549$ and NCl-HI 57 cells treated with nothing, the NC, miR-34c mimics or miR-34c mimics $+\mathrm{HMGBI}$, $* P<0.05$, $* * P<0.0 \mathrm{I}$ vs $\mathrm{NC}$ group, and $\# P<0.05$ vs mimics. (C and D) Upper panel: reactive oxygen species (ROS) production in A549 and NCl-HI57 cells treated with nothing, the NC, miR-34c mimics, or miR-34c mimics + HMGBI was detected using an ROS kit. Lower panel: the apoptosis rates of A549 and NCl-HI57 cells treated with nothing, the NC, miR-34c mimics or miR-34c mimics + HMGBI were evaluated by flow cytometric analysis, ${ }^{* * P}<0.01$ vs NC group, $\# P<0.05$ vs mimics. (E) Western blot analysis of Bax, $\mathrm{P}-\mathrm{ERK}$, elF2 $\boldsymbol{\alpha}$, GADDI53, and IREI $\boldsymbol{\alpha}$ expression in $\mathrm{A} 549$ and $\mathrm{NCl}-\mathrm{HI} 57$ cells treated with nothing, the NC, miR-34c mimics or miR-34c mimics + HMGBI. 


\section{Discussion}

NSCLC, characterized by large alterations in cellular phospholipid profiles, is the most common subtype of lung cancer derived from the bronchial epithelium. Although tremendous progress has been made in diagnostic and therapeutic technologies in recent decades, the incidence and prognosis of NSCLC remain dismal. ${ }^{25}$ Due to their tissueand time-specific expression patterns, miRNAs are considered to be promising biomarkers for use in the early detection of NSCLC. ${ }^{26}$ It is currently well known that miRNAs help to regulate the initiation and progression of NSCCL by directly regulating expression of oncogenes or tumor suppressors. For example, miR-671-3p was shown to suppress NSCLC progression by directly binding to and inhibiting Cyclin D2 (CCND2). ${ }^{27}$ Moreover, miR-19 was shown to promote NSCLC cell proliferation in vitro by binding to the 3'-UTR of CBX7 mRNA and thereby inhibiting CBX7 expression. ${ }^{28}$ Our study is the first to explore the roles and mechanisms of miR-34c in the pathogenesis of NSCLC, and the results not only increase our understanding of NSCLC pathogenesis, but also suggest a new potential therapeutic target for NSCLC.

ER stress can be induced by high rates of cell proliferation and metabolism, resulting in unfolded protein response (UPR) signaling cascades. Mounting evidence suggests that chemotherapeutic drug treatments, oncogene activation, B-Raf proto-oncogene mutations, H-Ras protooncogene mutations, and c-Myc amplification create ER stress in cancer cells. ${ }^{29}$ It was recently reported that modulation of ER stress could mediate the effects of multiple anti-cancer agents on NSCLC progression. ${ }^{30}$

HMGB1 is a small DNA-binding protein involved in various cellular processes. It is translocated from the nucleus into cytosol by either active or passive release processes. ${ }^{31}$ Accumulating evidence suggests that extracellular HMGB1 supports tumorigenesis by promoting inflammatory reactions, the epithelial-mesenchymal transition process, cell migration, and angiogenesis. ${ }^{32,33}$ The roles played by HMGB1 in regulating ER stress and ROS production have also been well documented. Cells exposed to external or internal stress release a series of damage-associated molecular patterns (DAMPs), including HMGB1; this release mediates an inflammatory response, ER stress, and ROS synthesis. ${ }^{18}$ Recent studies have shown that HMGB1 might affect the tumorigenesis of cancers by interacting with miRNAs. ${ }^{34,35}$ Furthermore, the association between miRNA expression and HMGB1 has been verified in various diseases. ${ }^{36}$ However, in this present study, we found that knockdown of HMGB1 remarkably inhibited NSCLC cell survival by promoting ER stress, ROS synthesis. This discrepancy might because of multifunction of ROS and ER stress in cancer. ${ }^{37,38}$

In summary, our findings revealed a significant downregulation of miR-34c and a significant upregulation of HMGB1 in NSCLC cells and tissues. Our functional assays demonstrated that miR-34c overexpression and HMGB1 knockdown significantly inhibited NSCLC progression and alleviated ER stress. Additionally, HMGB1 was identified as a target gene of miR$34 \mathrm{c}$, and we found that HMGB1 overexpression could abolish the effects of miR-34c mimics on NSCLC cell growth and ER stress in vitro. Our findings provide a novel molecular mechanism for NSCLC tumorigenesis, and suggest the miR-34c/ HMGB1axis as a promising therapeutic target for NSCLC.

\section{Acknowledgments}

This work was supported by the Shenzhen Healthcare Research Project (No.201604130027) and Shenzhen Health System Science Foundation (No.SZBC2018019).

\section{Disclosure}

The authors report no conflicts of interest in this work.

\section{References}

1. Torre LA, Siegel RL, Jemal A. Lung cancer statistics. Adv Exp Med Biol. 2016;893:1-19. doi:10.1007/978-3-319-24223-1_1

2. Qin H, Wang F, Liu H, et al. New advances in immunotherapy for non-small cell lung cancer. Am J Transl Res. 2018;10(8):2234-2245.

3. Szejniuk WM, Robles AI, McCulloch T, Falkmer UGI, Roe OD. Epigenetic predictive biomarkers for response or outcome to platinum-based chemotherapy in non-small cell lung cancer, current stateof-art. Pharmacogenomics J. 2018;19(1):5-14.

4. Lovly CM, Carbone DP. Lung cancer in 2010: one size does not fit all. Nat Rev Clin Oncol. 2011;8(2):68-70. doi:10.1038/ nrclinonc. 2010.224

5. Goldstraw P, Chansky K, Crowley J, et al. The IASLC lung cancer staging project: proposals for revision of the TNM stage groupings in the forthcoming (eighth) edition of the TNM classification for lung cancer. $J$ Thorac Oncol. 2016;11(1):39-51. doi:10.1016/j.jtho.2015.09.009

6. Buyukcelik A, Yalcin B, Utkan G. Multidisciplinary management of lung cancer. N Engl J Med. 2004;350(19):2008-2010; author reply 2008-2010.

7. Zarogoulidis K, Zarogoulidis P, Darwiche K, et al. Treatment of nonsmall cell lung cancer (NSCLC). J Thorac Dis. 2013;5 Suppl 4: S389-S396. doi:10.3978/j.issn.2072-1439.2013.07.10

8. Ambros V. The functions of animal microRNAs. Nature. 2004;431 (7006):350-355. doi:10.1038/nature02871

9. Misso G, Di Martino MT, De Rosa G, et al. MiR-34: a new weapon against cancer? Mol Ther Nucleic Acids. 2014;3:e194. doi:10.1038/mtna.2014.47

10. Xu L, Wang Q, Jiang W, Yu S, Zhang S. miR-34c ameliorates neuropathic pain by targeting NLRP3 in a mouse model of chronic constriction injury. Neuroscience. 2019;399:125-134. doi:10.1016/j. neuroscience.2018.12.030 
11. Xiao S, Li Y, Pan Q, et al. MiR-34c/SOX9 axis regulates the chemoresistance of ovarian cancer cell to cisplatin-based chemotherapy. J Cell Biochem. 2019;120(3):2940-2953. doi:10.1002/jcb.26865

12. Hu J, Hu X, Kan T. MiR-34c participates in diabetic corneal neuropathy via regulation of autophagy. Invest Ophthalmol Vis Sci. 2019;60(1):16-25. doi:10.1167/iovs.18-24968

13. Inagi R, Ishimoto $Y$, Nangaku M. Proteostasis in endoplasmic reticulum-new mechanisms in kidney disease. Nat Rev Nephrol. 2014;10 (7):369-378. doi:10.1038/nrneph.2014.67

14. Iurlaro R, Munoz-Pinedo C. Cell death induced by endoplasmic reticulum stress. Febs J. 2016;283(14):2640-2652. doi:10.1111/ febs. 13598

15. Wang M, Wang L, Guo Y, et al. A high mobility group box 1 (HMGB1) gene from Chlamys farreri and the DNA-binding ability and pro-inflammatory activity of its recombinant protein. Fish Shellfish Immunol. 2014;36(2):393-400. doi:10.1016/j.fsi.2013.12. 008

16. Shen J, Zhang J, Jiang X, Wang H, Pan G. LncRNA HOX transcript antisense RNA accelerated kidney injury induced by urine-derived sepsis through the miR-22/high mobility group box 1 pathway. Life Sci. 2018;210:185-191. doi:10.1016/j.lfs.2018.08.041

17. Wang S, Du S, Lv Y, Zhang F, Wang W. MicroRNA-665 inhibits the oncogenicity of retinoblastoma by directly targeting high-mobility group box 1 and inactivating the Wnt/beta-catenin pathway. Cancer Manag Res. 2019;11:3111-3123. doi:10.2147/CMAR.S200566

18. Dhivya Bharathi M, Justin-Thenmozhi A, Manivasagam T, et al. Amelioration of aluminum maltolate-induced inflammation and endoplasmic reticulum stress-mediated apoptosis by tannoid principles of emblica officinalis in neuronal cellular model. Neurotox Res. 2019;35 (2):318-330. doi:10.1007/s12640-018-9956-5

19. Zheng Y, Zhu G. HMGB1 suppresses colon carcinoma cell apoptosis triggered by coculture with dendritic cells via an ER stressassociated autophagy pathway. Mol Med Rep. 2018;17(2):3123-3132. doi:10.3892/mmr.2017.8202

20. Zhu M, Jiang Y, Wu H, et al. Gambogic acid shows anti-proliferative effects on Non-Small Cell Lung Cancer (NSCLC) cells by activating Reactive Oxygen Species (ROS)-induced Endoplasmic Reticulum (ER) stress-mediated apoptosis. Med Sci Monit. 2019;25:39833988. doi:10.12659/MSM.916835

21. Chou HL, Fong Y, Wei CK, et al. A quinone-containing compound enhances camptothecin-induced apoptosis of lung cancer through modulating endogenous ROS and ERK signaling. Arch Immunol Ther Exp (Warsz). 2017;65(3):241-252. doi:10.1007/s00005-0160424-8

22. El-Hussein A, Hamblin MR. ROS generation and DNA damage with photo-inactivation mediated by silver nanoparticles in lung cancer cell line. IET Nanobiotechnol. 2017;11(2):173-178. doi:10.1049/ietnbt.2015.0083

23. Yu Y, Tang D, Kang R. Oxidative stress-mediated HMGB1 biology. Front Physiol. 2015;6:93. doi:10.3389/fphys.2015.00098
24. Tong L, Chuang CC, Wu S, Zuo L. Reactive oxygen species in redox cancer therapy. Cancer Lett. 2015;367(1):18-25. doi:10.1016/j. canlet.2015.07.008

25. Osmani L, Askin F, Gabrielson E, Li QK. Current WHO guidelines and the critical role of immunohistochemical markers in the subclassification of non-small cell lung carcinoma (NSCLC): moving from targeted therapy to immunotherapy. Semin Cancer Biol. 2018;52(Pt 1):103-109. doi:10.1016/j.semcancer.2017.11.019

26. Arab A, Karimipoor M, Irani S, et al. Potential circulating miRNA signature for early detection of NSCLC. Cancer Genet. 2017;216217:150-158. doi:10.1016/j.cancergen.2017.07.006

27. Yao Y, Zhou Y, Fu X. miR6713p is downregulated in nonsmall cell lung cancer and inhibits cancer progression by directly targeting CCND2. Mol Med Rep. 2019. doi:10.3892/mmr.2019.9858

28. Peng X, Guan L, Gao B. miRNA-19 promotes non-small-cell lung cancer cell proliferation via inhibiting $\mathrm{CBX} 7$ expression. Onco Targets Ther. 2018;11:8865-8874. doi:10.2147/OTT.S181433

29. Alasiri G, Fan LY, Zona S, et al. ER stress and cancer: the FOXO forkhead transcription factor link. Mol Cell Endocrinol. 2018;462(Pt B):67-81. doi:10.1016/j.mce.2017.05.027

30. Chou CK, Liu W, Hong YJ, et al. Ethyl acetate extract of Scindapsus cf. hederaceus exerts the inhibitory bioactivity on human non-small cell lung cancer cells through modulating ER stress. Int J Mol Sci. 2018;19(7):1832. doi:10.3390/ijms 19071832

31. Sohun M, Shen H. The implication and potential applications of highmobility group box 1 protein in breast cancer. Ann Transl Med. 2016;4(11):217. doi:10.21037/atm.2016.04.05

32. Feng A, Tu Z, Yin B. The effect of HMGB1 on the clinicopathological and prognostic features of non-small cell lung cancer. Oncotarget. 2016;7(15):20507-20519. doi:10.18632/oncotarget.7050

33. Li Y, Tian J, Fu X, et al. Serum high mobility group box protein 1 as aclinical marker for ovarian cancer. Neoplasma. 2014;61(5):579-584. doi:10.4149/neo 2014070

34. Hou L, Chen J, Zheng Y, Wu C. Critical role of miR-155/FoxO1/ROS axis in the regulation of non-small cell lung carcinomas. Tumour Biol. 2016;37(4):5185-5192. doi:10.1007/s13277-015-4335-9

35. Wu YF, Ou CC, Chien PJ, Chang HY, Ko JL, Wang BY. Chidamideinduced ROS accumulation and miR-129-3p-dependent cell cycle arrest in non-small lung cancer cells. Phytomedicine. 2018;56:94102. doi:10.1016/j.phymed.2018.09.218

36. Yang L, Chai W, Wang Y, et al. Reactive oxygen species regulate the differentiation of acute promyelocytic leukemia cells through HMGB1mediated autophagy. Am J Cancer Res. 2015;5(2):714-725.

37. Kim C, Kim B. Anti-cancer natural products and their bioactive compounds inducing er stress-mediated apoptosis: a review. Nutrients. 2018;10(8):pii:E1021.

38. Martin KR, Barrett JC. Reactive oxygen species as double-edged swords in cellular processes: low-dose cell signaling versus highdose toxicity. Hum Exp Toxicol. 2002;21(2):71-75. doi:10.1191/ $0960327102 \mathrm{ht} 213 \mathrm{oa}$
OncoTargets and Therapy

Publish your work in this journal

OncoTargets and Therapy is an international, peer-reviewed, open access journal focusing on the pathological basis of all cancers potential targets for therapy and treatment protocols employed to improve the management of cancer patients. The journal also focuses on the impact of management programs and new therapeutic agents and protocols on patient perspectives such as quality of life, adherence and satisfaction. The manuscript management system is completely online and includes a very quick and fair peer-review system, which is all easy to use. Visit http://www.dovepress.com/ testimonials.php to read real quotes from published authors. 